\title{
Effectiveness of a summer school in influencing medical students' attitudes towards psychiatry
}

\author{
Sharon Beattie, ${ }^{1 \star}$ Clare Lister, $^{1 \star}$ Julie May Khan, ${ }^{1}$ Peter L. Cornwall ${ }^{1}$
}

The Psychiatrist (2013), 37, 367-371, doi: 10.1192/pb.bp.113.043513

${ }^{1}$ Tees, Esk and Wear Valleys NHS Foundation Trust, Teeside

Correspondence to Clare Lister (clare.lister@nhs.net)

First received 26 Mar 2013, accepted 4 Apr 2013

\begin{abstract}
Aims and method Summer schools are advocated as part of the national recruitment initiative despite little evidence of their impact. This study evaluates the effectiveness of a 3-day non-clinical initiative. Change in attitudes and career intention were measured by administering a questionnaire, which included the 30-item Attitudes Toward Psychiatry (ATP-30) survey, at the start and end of the event.
\end{abstract}

Results Mean ATP-30 scores increased from 119 to 128, which represented a highly statistically significant change $(t=5.40$, d.f. $=18, P<0.001)$. A positive shift in intention to pursue psychiatry as a career was demonstrated.

Clinical implications These results suggest well-planned summer schools can have a significant impact on students' attitudes. Despite high initial ATP-30 scores a positive shift in attitudes and career intentions was still seen. Further evaluation of the longitudinal impact is needed. Events such as this are important and likely produce a cumulative effect alongside other recruitment strategies.

Declaration of interest None.
Recruitment of doctors into psychiatry has been a concern for decades. ${ }^{1}$ In more recent years the issue of recruitment has again become topical as psychiatry has consistently failed to fill all its training posts nationally. In 2011 and 2012 , only $83 \%$ and $85.3 \%$ of core training year 1 (CT1) posts respectively were filled following the second rounds of recruitment. $^{2,3}$ This raises many issues including concerns about future workforce planning and also that a need to fill training posts for service provision purposes may lead to recruitment of a lower calibre of trainees with longer-term consequences for the specialty. ${ }^{4}$ The Royal College of Psychiatrists has therefore implemented a national 5-year recruitment campaign, ${ }^{2}$ with its primary aim to increase recruitment to the $\mathrm{CTl}$ grade of core psychiatry training, achieving a $50 \%$ increase in applications and a $95 \%$ fill rate by the end of the campaign. The College recruitment strategy outlines a variety of ways in which it is hoped that this will be achieved. One of these includes the creation of summer schools in each regional division, in the hope that this may 'seed the idea of psychiatry as a career'.

In the UK, psychiatry summer schools are a relatively recent phenomenon. They were introduced by the Royal College of Psychiatrists and the Institute of Psychiatry in 2009 when the first event was held in London. Since then this event has run annually, and the concept has been replicated with summer schools emerging in other centres in the UK. Despite much time, energy and effort being

*These authors contributed equally to the work invested in such endeavours, there has been very little published on the outcomes of these events; including what makes a successful event and the impact it has on the medical students who attend. A recent article published in The Psychiatrist helpfully outlines guidance on how to create a summer school based on the authors' experience but recognises there is a need for evaluation of such events. ${ }^{5}$

Concerns about recruitment of medical students into psychiatry are not unique to the UK. Similar concerns have been noted in the USA and Canada. Such issues led to the development of a summer school in Toronto that was replicated in other regions in Canada. ${ }^{6}$ This Canadian enterprise began in 1994 and data from its evaluation suggest a positive influence on career intentions, although less than half the students attending were ultimately matched into psychiatric residency programmes. Interestingly the success rate was higher when only students who attended the parent event were considered. Confounding factors such as difference in summer school programmes and the impact of other recruitment strategies may have contributed to this effect.

In creating a summer school it is important to consider the issues that may influence medical students' attitudes towards psychiatry. There have been many articles published exploring the reasons why medical students and junior doctors are deterred from pursuing psychiatry as a career. Factors identified include a perceived lack of scientific basis for psychiatric practice, that psychiatry is removed from 'real medicine', the perception that patients do not improve or get better, students' concerns about overidentifying with patients and the perceived low status 
of psychiatry within the profession. ${ }^{1,7-14}$ We were also aware from observations and local unpublished work that even following medical school psychiatric placements students are often not aware of the multiple career strands within psychiatry. They also sometimes struggle to appreciate the role of the psychiatrist within patient journeys, and so cannot identify with the reality of what a career as a psychiatrist may entail.

\section{Summer school programme}

Tees, Esk and Wear Valleys NHS Foundation Trust ran their first summer school in July 2012 designed by the authors of this article. When devising the programme the team spent time seeking clarity of the purpose of the summer school, which was encompassed in our aims and objectives. The vision was to create an event that did not replicate medical school experience but that aimed to specifically target factors known to negatively influence students and buffer against these, in addition to also nurturing interested students by including items that are felt to attract students towards psychiatry. We aimed to positively influence students' attitudes and communicate psychiatry as a stimulating and challenging yet rewarding specialty that requires compassionate and skilled doctors who have an important role in assessing and managing patients. The target audience were medical students with an interest in psychiatry, but who had not decided on their career trajectory. Although clinical experience is vital in positively influencing medical student attitudes, it was felt that within a 3-day event we were unlikely to be able to incorporate any meaningful patient content without compromising other aspects of the programme. We aimed to evaluate this event through measuring its impact on students' career intentions and attitudes towards psychiatry.

Each item within the programme (see online Appendix DS1) was included for the value it could add in communicating a positive message about careers in psychiatry. We were particularly careful to ensure that we highlighted the role of the doctor, the positive challenge involved in assessing and managing cases of complexity and diagnostic uncertainty, the privilege and importance of the doctor-patient experience and a sense of therapeutic optimism through recovery. We selectively involved clinicians who could contribute not only their expertise but who also communicated enthusiasm and professionalism, bearing in mind that the recruitment literature highlights the importance of positive role modelling. ${ }^{4,15}$ We also focused on allowing the students opportunities to build relationships with the clinicians involved in the event. Aware that our programme did not involve actual patient contact, there was a focus on bringing clinical material to life without the need to attend the clinical environment.

\section{Method}

All summer school delegates $(n=19)$ were asked to complete a questionnaire at the beginning and end of the event. The questionnaire included basic demographics, questions about career intentions, the 30-item Attitudes Toward Psychiatry
$(\mathrm{ATP}-30)^{16}$ scale and qualitative free-text feedback on every session in the programme.

The ATP-30 is an attitudinal survey designed to look at medical students' attitudes towards psychiatry. It was created in 1982 by Burra et $a l,{ }^{16}$ and has not been updated since this time but has been used in numerous studies. The scale was shown to be sensitive in detecting positive shifts in attitude after students had clinical psychiatric placements. In the original paper the third- and fourth-year medical students' baseline ATP-30 score was a mean of 104.2, with an increase after clinical exposure to 108.5. Since then the scale has been used internationally to assess the impact of teaching, clinical attachments and promotional strategies. ${ }^{17-19}$ On completing the questionnaire participants are asked to respond to 30 statements using a 5-point likert scale (strongly agree to strongly disagree). Statements are equally positively or negatively phrased. For each respondent a total attitudinal score is calculated. The ATP-30 does not have proven validity for specific items within the scale, but previous research has looked in more detail at the scores and changes of scores on various items to highlight perhaps more subtle influences. ${ }^{20}$ We have therefore also looked at this within our analysis.

We administered the questionnaire using TurningPoint (version 4.2.4.1012 Capacity 5, which is an interactive audience participation software programme that is integrated within Powerpoint 2007, using Windows XP Professional. Students were allocated a number to track changes in their attitudes while maintaining anonymity. Embedding the questionnaire within the opening and closing sessions of the event ensured a $100 \%$ response rate. We then planned to compare the mean ATP-30 scores using a paired $t$-test.

\section{Results}

\section{Participants}

The summer school was attended by 19 students from various medical schools across the country (Table 1). They ranged in age from 19 to 35 and the majority (74\%, $n=14$ ) were female. All attended UK medical schools and the majority $(89 \%, n=17)$ spoke English as their first language.

\section{Attitudes towards psychiatry}

The ATP-30 scores showed that our summer school students already had relatively high baseline scores demonstrating positive attitudes towards psychiatry. The mean ATP-30 score before the summer school was 119 (s.d.=9.8). Despite these positive initial attitudes, the students were not certain of their career intentions, with only $47 \%$ definitely or probably indicating their intent to pursue psychiatry as a career prior to the summer school. There was a notable increase in mean ATP-30 score after the summer school, by 9 points, to a total of 128 (s.d. $=10.6$, Fig. 1).

As the results were normally distributed we used a paired $t$-test to compare the ATP-30 mean difference scores before (119) and after (128) the event. Despite the high baseline scores we demonstrated a highly statistically 


\begin{tabular}{lc} 
Table 1 Demographic data & \\
Variable & $n(\%)$ \\
\hline Gender & \\
Male & $5(26)$ \\
Female & $14(74)$ \\
\hline Medical school year & \\
First & $4(21)$ \\
Second & $10(53)$ \\
Third & $5(26)$ \\
\hline Age, years & \\
$\leqslant 20$ & $6(31)$ \\
$21-25$ & $10(53)$ \\
$26-30$ & $2(11)$ \\
$31-35$ & $1(5)$ \\
$\geqslant 36$ & $0(0)$ \\
\hline First language & \\
English & $17(89)$ \\
Other & $2(11)$ \\
\hline Country of birth & \\
UK & $15(79)$ \\
Ireland & $1(5)$ \\
Other & $3(16)$ \\
&
\end{tabular}

significant positive increase in attitudes towards psychiatry $(t=5.40$, d.f. $=18, P<0.001)$ following the event.

We also demonstrated a positive shift in career intention with an increase to $68 \%(n=13)$ definitely or probably wanting to pursue psychiatry as a career. At the end of the event none of the students had rejected psychiatry nor did any remain unsure whether to consider it as a potential career (Fig. 2).

\section{Details of response}

We looked closely at areas within the ATP-30 to analyse potential specific influences of our programme. We note that the ATP-30, as mentioned previously, does not have proven validity for specific items, but we feel this was an important way to break down our results further, and this has been done previously within the literature. It is important to note that none of the 30 attitudinal statements showed a decrease in score from baseline. They all either remained

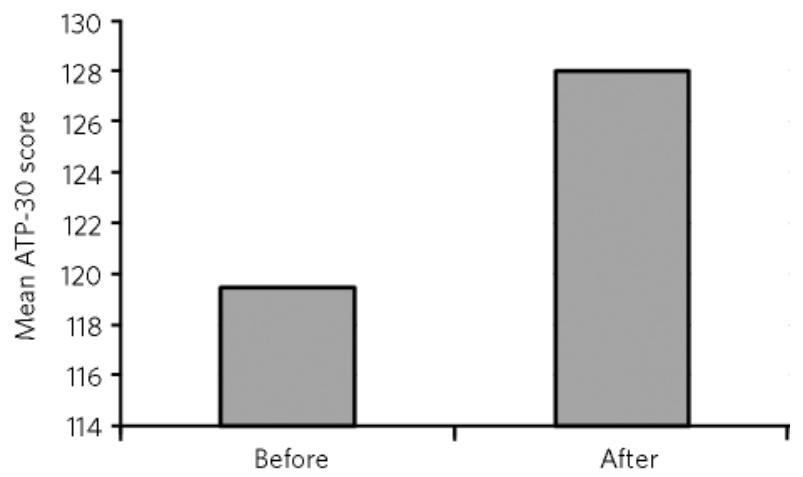

Fig 1 Mean Attitudes Toward Psychiatry (ATP-30) scores at the beginning and end of the summer school. at the same level or shifted, becoming more positive towards psychiatry.

\section{Items with the lowest baseline scores}

(a) These days psychiatry is the most important part of the curriculum in medical schools.

(b) The majority of students report that their psychiatric undergraduate training has been very valuable.

(c) Psychiatry is a respected branch of medicine.

This appears to show that although most students at our summer school were in their preclinical years, they were still unsure about psychiatry's place in medical school and the medical arena as a whole.

\section{Items with the highest baseline scores}

(a) Psychiatric illness deserves at least as much attention as physical illness.

(b) It is interesting to try and unravel the causes of a psychiatric illness.

(c) The practice of psychiatry allows the development of really rewarding relationships with people.

Before the summer school, as expected, those areas scoring highest revealed an inherent interest in some of the factors that make psychiatry unique.

\section{Items with the largest increase in score}

(a) It is quite easy for me to accept the efficacy of psychiatry.

(b) Psychiatry is a respected branch of medicine.

(c) Psychiatry has very little scientific information to go on.

(d) Psychiatrists tend to be at least as stable as the average doctor.

(e) Psychiatric patients are often more interesting to work with than other patients.

Results suggest that our programme seemed to have a particular influence on the perception of the specialty's standing in medicine and as a scientific discipline.

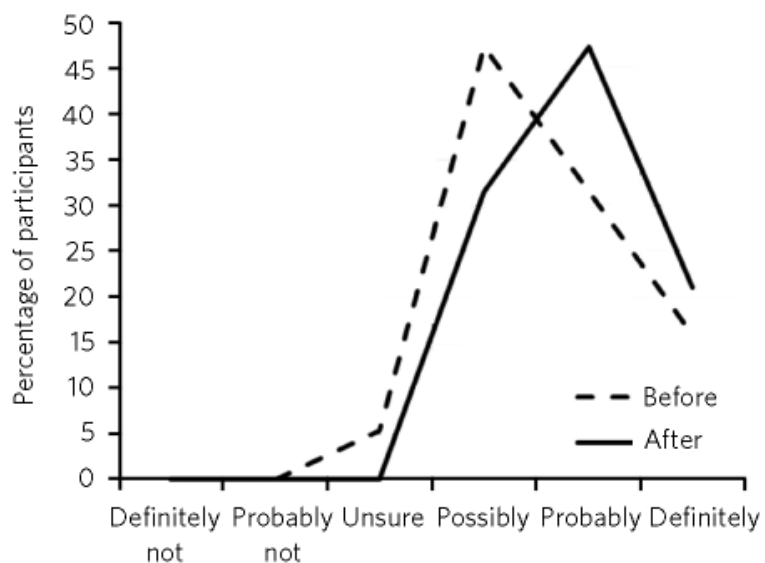

Fig 2 Participants' answers regarding choosing psychiatry as a career at the beginning and end of summer school. 
Items with no change in score

(a) People taking up psychiatry are running away from real medicine.

(b) It is interesting to try and unravel the causes of a psychiatric illness.

(c) The practice of psychiatry allows the development of really rewarding relationships with people.

(d) Psychiatrists talk a lot but do very little.

(e) The practice of psychotherapy basically is fraudulent since there is no strong evidence that it is effective.

The majority of areas that the summer school did not influence already had high scores at baseline, although results suggest that there was a potential weakness in our programme in looking at addressing perceptions regarding psychotherapy.

\section{Discussion}

These results suggest that a non-clinical summer school positively portraying psychiatry can have a significant impact on medical students' attitudes towards the specialty. The mean baseline ATP-30 score of 119 (s.d.=9.8) in our sample of students is higher than baseline scores seen in other medical student populations. For example the mean baseline ATP-30 for fourth-year medical students in one study was 102.6 (s.d. $=10.1)^{13}$ and in another study sixthform school pupils had a mean baseline of 110 (s.d. = 12.8). ${ }^{21}$ We were surprised that in spite of their initial high ATP-30 scores this short intervention still produced a significant positive shift in attitude and had an influence on students' apparent career intentions.

We feel this is evidence that summer schools can be effective in influencing students' opinions. Previous research has suggested that clinical encounters are pivotal in influencing students and changing career intentions. ${ }^{10}$ However, our carefully designed programme suggests that patient contact in this type of event is not essential. We believe this is because the programme compensated by focusing on bringing to life patient experiences through cases, highlighting recovery, emphasising an empathic approach and having workshops that included case studies that embodied the role of the psychiatrist in patient journeys. Through not including face-to-face clinical time the programme was able to focus and target areas known to deflect students from psychiatry.

We also found attitudes were changed to a varying degree, and feel this shows that the emphasis of the summer school programme is important. There are already identified aspects, so called 'push factors', which make psychiatry less appealing and our baseline scores highlighted one of these especially, regarding psychiatry's portrayal within the wider medical landscape. It is important to tailor programmes to have an impact on areas where we are aware there are frequently negative misconceptions among students, and that we know are important in discouraging them from pursuing psychiatry. We have shown that developing a carefully chosen programme of events to do just this has led to these areas being influenced substantially more. This was demonstrated through aspects of attitude such as respect for psychiatry, its scientific basis, efficacy of treatment and the interesting nature of work within psychiatry increasing more than others. We also have learnt that having less focus on psychotherapy and talking therapies in general has meant that we did not create opportunities to positively change students' opinions, and therefore did not seem to influence this aspect on evaluation. It is important to note that although there were a few items on the ATP-30 where the score did not increase, there were no areas of the students' attitudes that we appear to have had a negative impact on.

\section{Implications}

Further evaluation of the longitudinal impact of such interventions needs to be explored, in order to see whether such attitudinal changes are sustained. There is evidence in previous studies ${ }^{11}$ that attitudes are subject to erosion and alteration at specific points of undergraduate and postgraduate training. This is especially true when considering the stage of training of the delegates, none of whom were in the final years of medical training.

Consideration needs to be given to strategies where the positive experiences of summer schools can be reinforced throughout their medical training. This is an important early influence and contact point. It may be that summer school reunions, using social media to form networks or more formal mentor arrangements could sustain the positive influence over time. We feel summer schools are important and play a part alongside other positive undergraduate experiences of the specialty. We believe they can produce a cumulative effect on influencing students' positive intentions to pursue psychiatry as a career and that summer schools have a legitimate part to play in the recruitment strategy.

\section{About the authors}

Dr Sharon Beattie is a specialty trainee year 5 in general adult psychiatry in Tees, Esk and Wear Valleys NHS Foundation Trust. Dr Clare Lister is a core trainee year 2 in psychiatry in Tees, Esk and Wear Valleys NHS Foundation Trust. Ms Julie May Khan is the medical education manager for Tees, Esk and Wear Valleys NHS Foundation Trust. Dr Peter L. Cornwall is deputy medical director for Tees and was previously the director of medical education for Tees, Esk and Wear Valleys NHS Foundation Trust.

\section{References}

1 Goldacre MJ, Turner G, Fazel S, Lambert T. Career choices for psychiatry: national surveys of graduates of 1974-2000 from UK medical schools. Br J Psychiatry 2005; 186: 158-64

2 Royal College of Psychiatrists. Recruitment Strategy 2011-2016. Royal College of Psychiatrists, 2012 (http://www.rcpsych.ac.uk/pdf/ Recruitment\%20Strategy\%20-\%2010092013.pdf).

3 Royal College of Psychiatrists. CT1 Outcomes and Competition - 2012. Royal College of Psychiatrists, 2012 (http://www.rcpsych.ac.uk/pdf/ CT1\%20fill\%20rate-10.09.2012.pdf).

4 Pidd SA. Recruiting and retaining psychiatrists. Adv Psychiatr Treat 2003; 9: 405-11.

5 Greening J, Tarn M, Purkis J. How to run a psychiatry summer school. Psychiatrist 2013; 37: 65-71.

6 Andermann L, De Souza C, Lofchy J. The psychiatry institute for medical students: a decade of success. Acad Psychiatry 2010; 34: 2. 
7 Goldacre MJ, Fazel S, Smith F, Lambert T. Choice and rejection of psychiatry as a career: surveys of UK medical graduates from 1974 to 2009. Br J Psychiatry 2013; 202: 228-34.

8 Dein K, Livingston G, Bench C. 'Why did I become a psychiatrist?': survey of consultant psychiatrists. Psychiatr Bull 2007; 31: 227-30

9 Brown TM, Addie K, Eagles JM. Recruitment into psychiatry: views of consultants in Scotland. Psychiatr Bull 2007; 31: 411-3.

10 Manassis K, Katz M, Lofchy J, Wiesenthal S. Choosing a career in psychiatry: influential factors within a medical school program. Acad Psychiatry 2006; 30: 325-9.

11 Maidment R, Livingston G, Katona C, McParland M, Noble L. Change in attitudes to psychiatry and intention to pursue psychiatry as a career in newly qualified doctors: a follow-up of two cohorts of medical students. Med Teach 2004; 26: 565-9.

12 Eagles JM, Wilson S, Murdoch JM, Brown T. What impact do undergraduate experiences have upon recruitment into psychiatry? Psychiatr Bull 2007; 31: 70-2.

13 McParland M, Noble L, Livingston G, McManus C. The effect of a psychiatric attachment on students' attitudes to and the intention to pursue psychiatry as a career. Med Educ 2003; 37: 447-54.

14 Lambert TW, Turner G, Fazel S, Goldacre MJ. Reasons why some UK medical graduates who initially choose psychiatry do not pursue it as a long-term career. Psychol Med 2006; 36: 679-84.
15 Wear D, Skillicorn J. Hidden in plain sight: the formal, informal, and hidden curricula of a psychiatry clerkship. Acad Med 2009; 84: 451-8.

16 Burra P, Kalin R, Leichner P, Waldron JJ, Handsforth JR, Jarrett FJ, et al. The ATP 30 - a scale for measuring medical students' attitudes to psychiatry. Med Educ 1982; 16: 31-8.

17 Kuhnigk O, Hormann M, Bothern AM, Haufs C, Bullinger M, Harendza S. Influence of educational programs on attitudes of medical students towards psychiatry: effects of psychiatric experience, gender, and personality dimensions. Med Teach 2009; 31: e303-10.

18 Singh SP, Baxter $\mathrm{H}$, Standen $\mathrm{P}$, Duggan $\mathrm{C}$. Changing the attitudes of 'tomorrow's doctors' towards mental illness and psychiatry: a comparison of two teaching methods. Med Educ 1998; 32: 115-20.

19 Agbayewa MO, Leuchner PP. Effects of a psychiatric rotation on psychiatric knowledge and attitudes towards psychiatry in rotating interns. Can J Psychiatry 1985; 30: 602-4.

20 Thompson C, Dogra N, McKinley R. Survey of general practitioners' attitudes towards psychiatry. Psychiatrist 2010; 34: 525-8.

21 Maidment R, Livingston G, Katona M, Whitaker E, Katona C. Carry on shrinking: career intentions and attitudes to psychiatry of prospective medical students. Psychiatrist 2003; 27: 30-2. 